\title{
Electronic Properties and Charge Transfer of Topologically Protected States in Hybrid Bismuthene Layers
}

\author{
Andreia L. da Rosa,* Erika Nascimento Lima,* Maurício Chagas da Silva,* Renato Borges Pontes,* \\ Jailton Souza de Almeida,* Tome Mauro Schmidt,* and Thomas Frauenheim*
}

Cite This: J. Phys. Chem. C 2020, 124, 11708-11715

Read Online

ACCESS | Llll Metrics \& More | 回 Article Recommendations

ABSTRACT: We have performed first-principles calculations of electronic and dielectric properties of single-layer bismuth (bismuthene) adsorbed with $-\mathrm{COOH}$ groups. We show that in a high coverage regime, the $\mathrm{Bi}-\mathrm{COOH}$ hybrid structure is a two-dimensional topological insulator with protected edge Dirac states. The adsorption process of $-\mathrm{COOH}$ induces a planar configuration to the initially pristine buckled bismuthene. We claim that the stability of these planar structures mainly stems from strain induced by the adsorption of the $-\mathrm{COOH}$ organic group, but it is also related to ligand-ligand interactions. Furthermore, we demonstrate that many-body corrections are crucial to obtain a proper description of the electronic and dielectric properties of the investigated hybrid systems. Analysis of the charge density shows that the role of this organic group is not only to stabilize the layer but also to functionalize it, which is very important for future applications such as sensing and biomolecules immobilization, as well as in electronic spintronic and even optical devices.

\section{INTRODUCTION}

Only recently has the existence of ultrathin bismuth layers been experimentally confirmed, either synthesized on a silicon carbide substrate $^{1}$ or as free-standing layers. ${ }^{2}$ Such bismuth-based nanostructures when composed of double layers were previously theoretically predicted to have topological insulator behavior with protected nontrivial Dirac states. ${ }^{3-6}$ These layers were found to be robust against perturbations that preserve time reversal symmetry, such as strain and applied external electric field. ${ }^{7}$ Optical properties of bismuthene using many-body methods have been recently reported by Kecik et al. ${ }^{8}$ A strong absorption peak around $2.2 \mathrm{eV}$ is predicted, with an exciton with binding energy of $0.18 \mathrm{eV}$. This indicates that bismuthene could be useful for applications in solar cells and light-emitting devices. However, adsorption of small ligands on bismuthene suggests that it preserves its topological insulator behavior., ${ }^{3,4,9}$

Theoretical predictions show that valley-polarized quantum anomalous Hall states emerge as bismuthene are adsorbed with hydrogen, ${ }^{10}$ enlarging its band gap compared to bismuthene layers and indicating that ligand-mediated interactions with the substrate may be important. Moreover, it has been proposed that $-\mathrm{COOH}$ groups can induce ferroelectric behavior on bismuthene. 9 This suggests that ligand adsorption on bismuthene may affect not only its electronic structure but also its reactivity, dielectric, and optical properties. Despite all that, the role of organic ligands on introducing new functionalities to bismuthene, such as change of reactivity and tunable optical properties, remains largely unexplored. This is a crucial requirement for further applications such as immobilization of organic or biomolecules and also in the field of catalysis.

In this work, we employ density-functional theory (DFT) and the $G W$ method to show that the bismuthene-based hybrid structures have topological insulating behavior. We show that the adsorption of $-\mathrm{COOH}$ groups induces a planar geometry on the initially buckled bismuthene structure. Most importantly, our results reveal that $-\mathrm{COOH}$ also introduces a new function to bismuthene by changing the reactivity of the hybrid system. Finally, we perform $G W$ calculations to obtain the electronic band gap and dielectric properties of the hybrid layers.

\section{METHODOLOGY}

The electronic structure and chemical bond analysis of bismuthene adsorbed with $-\mathrm{COOH}$ groups were investigated within density-functional theory framework. The projected augmented wave method $(\mathrm{PAW})^{11,12}$ combined with the generalized gradient approximation (GGA) ${ }^{13}$ as implemented in the VASP package has been employed. ${ }^{12,14}$ The lattice parameters and atomic positions were fully relaxed until the atomic forces converge to less than $10^{-4} \mathrm{eV} / \AA$. A vacuum region of at least $10 \AA$ perpendicular to the bismuthene layer was

Received: March 17, 2020

Revised: April 25, 2020

Published: April 30, 2020

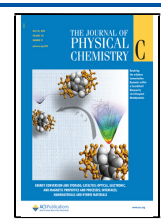


introduced to avoid interaction between atoms in neighboring cells. The reciprocal space was sampled with a $(10 \times 10 \times 1) \mathbf{k}$ point $\Gamma$-centered grid. The Kohn-Sham wave functions were expanded in a plane-wave basis set with an energy cutoff of 500 $\mathrm{eV}$. Spin-orbit coupling (SOC) was included in all calculations, including relaxation, electronic structure, and dielectric properties. The nature of the chemical bonds in the hybrid system was investigated by calculating the charge density difference and electron localization function (ELF). The dielectric function of the hybrid system was calculated using the $G W$ approximation with eight iterations in the Green's function $G$, which was found to be sufficient to converge the quasi-particle eigenvalues with respect to $G_{0} W_{0}$ calculations. In the later case, a $(5 \times 5 \times 1) \mathbf{k}$ point grid was used due to the high computational costs. Tests with up to $(12 \times 12 \times 1) \mathrm{k}$-points for bismuthene have shown that a smaller set is enough to achieve convergence. Moreover, due to high computational costs, the energy cutoff for $G W$ calculations has been decreased to $400 \mathrm{eV}$.

\section{RESULTS AND DISCUSSION}

The stability of the pristine buckled and the planar bismuthene has been investigated by varying the lattice parameters $a$ and $b$ and fully relaxing all the atoms in the unit cell. The optimized inplane lattice parameters are $a=b=4.39 \AA$ for the buckled structure and $a=b=5.30 \AA$ for the planar one, as shown in Figure 1. Our DFT-GGA calculated structural parameters for buckled bismuthene are also in very good agreement with previous theoretical and experimental values in the literature. $^{15-19}$

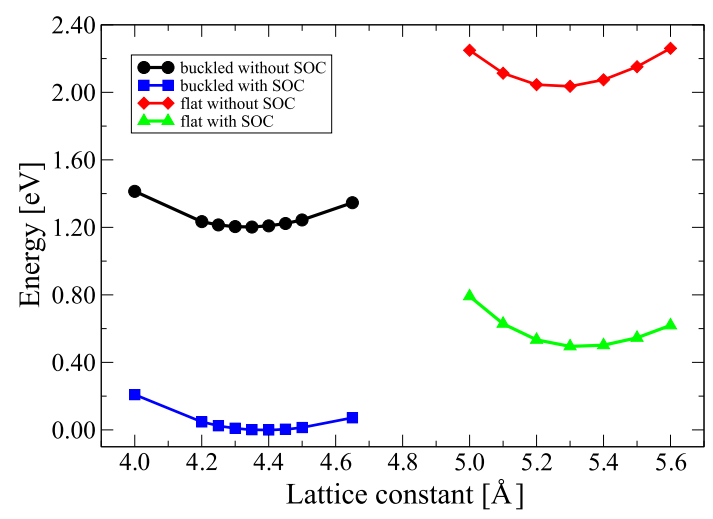

Figure 1. Total energy as a function of in-plane lattice constant of buckled and planar bismuthene with and without the inclusion of SOC. The zero of energy is set to the most stable structure.

We can see from Figure 1 that the inclusion of SOC does not change significantly the lattice parameters but lowers the total energy of bismuthene. We found that the buckled bismuthene structure is much more stable than the planar configuration with an energy difference of $1.55 \mathrm{eV}(1.20 \mathrm{eV})$ with (without) the inclusion of SOC. The structural stability of buckled bismuthene compared to the planar one can be understood considering that in two-dimensional bismuthene each bismuth atom has three nearest neighbors forming $\sigma$-like bonds. This structural configuration can be explained by noticing that the electronic density of localized bonds and/or lone electrons pairs seek for spatial configuration which minimizes the electron-electron interactions. This means that the angles between the localized bonds and/or lone electrons pair should be greatest to minimize the repulsion between them. The $\mathrm{sp}^{3}$ hybridization presents an angle of about $109^{\circ}$ between the lone electrons pairs of $\mathrm{Bi}$ centers and the $\mathrm{Bi}-\mathrm{Bi} \sigma$ bonds. This geometric arrangement has an angle that is larger than the $90^{\circ}$ considering a sp ${ }^{2}$ hybridization. This minimizes the electron-electron interactions between the $\mathrm{Bi}-\mathrm{Bi} \sigma$-like bond and the nonbonded lone electrons pairs of $\mathrm{Bi}$ centers producing a buckling of $1.72 \AA$, which is in agreement with Freitas et al. ${ }^{4}$ In order to achieve the structural stability, the out-of-plane $\left(\mathrm{sp}^{3}\right.$-like) bonds are favored with respect to the planar ( $\mathrm{sp}^{2}$-like) bonds, leading to a buckled structure.

In order to functionalize bismuthene, we have adsorbed $-\mathrm{COOH}$ on one $(1 / 2 \mathrm{ML})$ and on both sides ( $1 \mathrm{ML})$. The ligands are adsorbed on top positions of bismuth atoms on both sides of the bismuth surface. We found that the configurations where the radicals are on top positions are more stable than hollow or bridge sites. Interestingly enough, both bridge and hollow configurations spontaneously relax to a configuration where $-\mathrm{COOH}$ groups are sitting at top positions. Although we cannot rule out that smaller coverages may be present, we should point out that lower coverages were found to yield less stable structures in other two-dimensional hybrid systems, such as germanene. $^{20}$

Figure 2 shows top and side views of both relaxed and hybrid bismuthene. It is important to mention that a planar configuration for bismuthene can be experimentally obtained by applying external strain or simply by preparing bismuth layers on a suitable substrate. No buckling exists for planar bismuthene, as shown in Figure 2a. However, buckled (corrugated) bismuthene, as shown in Figure $2 \mathrm{~b}$, has a buckling of $1.60 \AA$, in good agreement with refs 3 and 4 . A configuration with $1 / 2$ $\mathrm{ML}$ of $-\mathrm{COOH}$ groups adsorbed on bismuthene could be experimentally realized on bismuth on top of a substrate, for example. This structure shown in Figure $2 c$ has an in-plane lattice parameter equal to $5.44 \AA$. This hybrid system relaxes to an almost perfect planar structure, with buckling of $0.03 \AA$. Upon adsorption of $1 \mathrm{ML}$ of $-\mathrm{COOH}$ groups, the initial buckled geometry of bismuthene also relaxes to a near planar structure, with an in-plane lattice parameter of $5.10 \AA$, as shown in Figure $2 \mathrm{~d}$. There are two main driving forces for the planarity of these structures: the ligand-ligand interaction and the electronegativity of the ligand. The $\mathrm{sp}^{2}$-like hybridization should be preferred in the adsorbed layers against the $\mathrm{sp}^{3}$-like in buckled bismuthene. As a consequence of these additional ligand-ligand interactions, the system assumes a quasi-planar configuration that increases the in-planar lattice constant. The $\mathrm{Bi}-\mathrm{Bi}$ bonds are now larger if compared to buckled bismuthene and the $\mathrm{Bi}-$ $\mathrm{Bi}-\mathrm{C}$ angle is reduced to approximately $82^{\circ}$ in a $\mathrm{sp}^{2}$-like hybridization, as shown in Figure 2. A similar behavior has been suggested for organic functionalized germanene. ${ }^{20}$

Figure 3 shows the orbital projected band structure for bismuthene with planar and buckled structures and also upon adsorption of $-\mathrm{COOH}$ groups at $1 / 2$ (one side) and 1 (both sides) ML coverage. In Figure $3 \mathrm{a}$ one can see that the bismuth $\mathrm{p}_{x}, \mathrm{p}_{y}$, and $\mathrm{p}_{z}$ orbitals dominate the bands near the Fermi level with little contribution from s orbitals for the planar structure shown in Figure 2a. A direct band gap of $0.8 \mathrm{eV}$ is found at the $\mathrm{M}$ point. In Figure $3 b$ one can see the Rashba splitting at $\Gamma^{3}$ for the buckled structure shown in Figure $2 \mathrm{~b}$. The sum of $\mathrm{p}_{x}$ and $\mathrm{p}_{y}$ and $\mathrm{p}_{z}$ bismuth orbitals has a similar contribution to the bands near the Fermi level with, again, little contribution from s orbitals. While $\mathrm{p}_{x}$ and $\mathrm{p}_{y}$ dominate the conduction band minimum $(\mathrm{CBM}), \mathrm{p}_{z}$ bismuth orbitals dominate the valence band maximum (VBM). An indirect band gap of $0.45 \mathrm{eV}$ is found. 

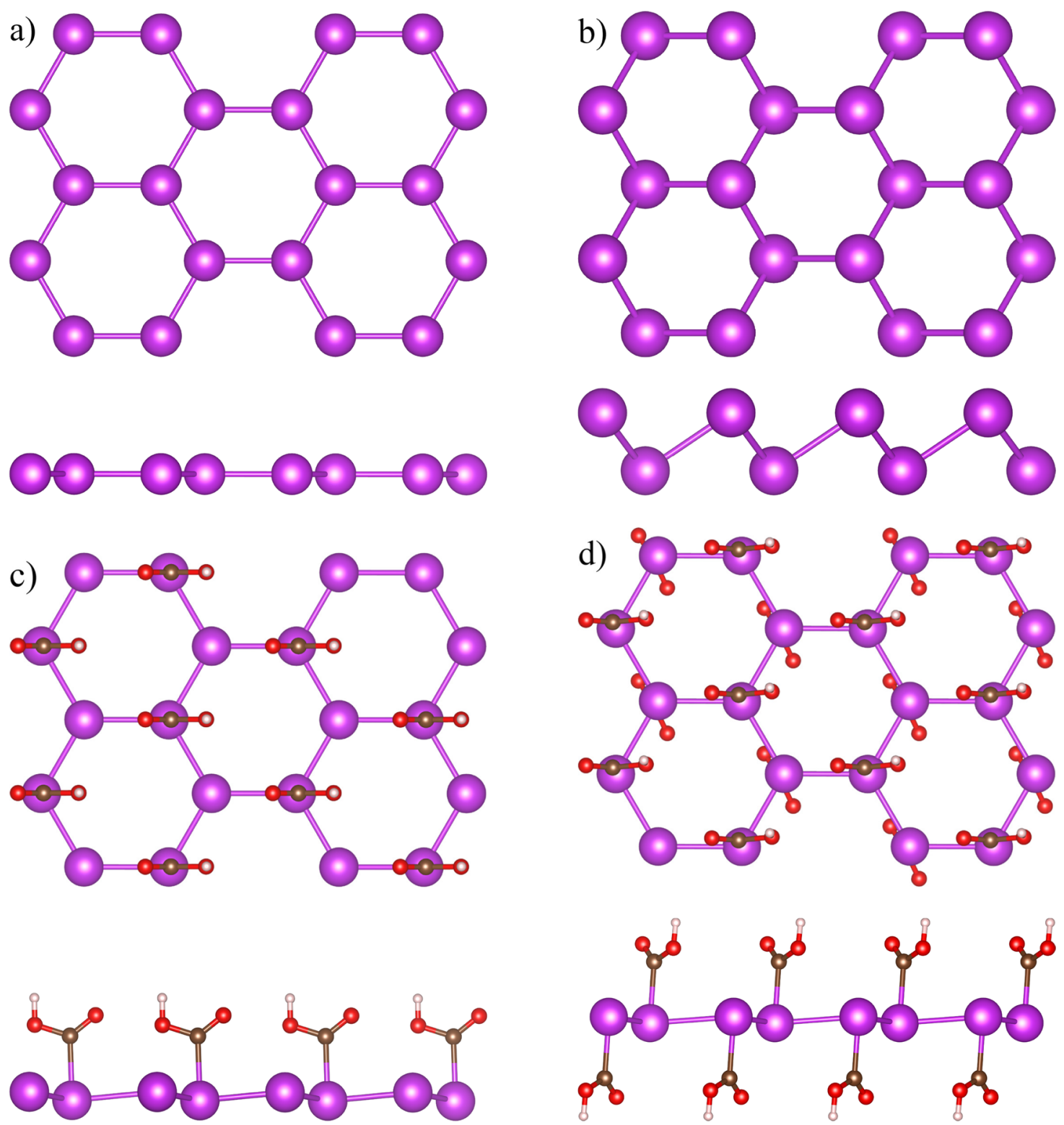

Figure 2. Top and side views of (a) planar bismuthene, (b) buckled bismuthene, and (c) bismuthene adsorbed with $-\mathrm{COOH}$ in the $1 / 2 \mathrm{ML}$ (one side) and (d) in the $1 \mathrm{ML}$ (both sides) coverage regime. Magenta, red, brown, and white spheres stand for bismuth, oxygen, carbon, and hydrogen atoms, respectively.

The band structure, shown in Figure 3, projected on bismuth atoms (c) and on $-\mathrm{COOH}$ atoms (d) upon $-\mathrm{COOH}$ adsorption on bismuthene in the $1 / 2 \mathrm{ML}$ coverage regime shows that $\mathrm{p}_{x}, \mathrm{p}_{y}$, and $\mathrm{p}_{z}$ orbitals dominate the bands near the Fermi level. The system exhibits a metallic behavior with bands belonging to the unsaturated bismuth atoms contributing mostly to states close to the Fermi level.

From the orbital projected band structure in Figures $3 \mathrm{e}, \mathrm{f}$, one can see that the $\mathrm{p}_{x}, \mathrm{p}_{y}$, and $\mathrm{p}_{z}$ orbitals dominate the bands near the Fermi level. These states stem mainly from bismuth atoms. The change in the geometry upon $-\mathrm{COOH}$ adsorption shown in Figure $3 \mathrm{e}, \mathrm{f}$ shifts the conduction band minimum (CBM) from $\mathrm{K}$ to $\Gamma$. We can see contributions from the $-\mathrm{COOH}$ group at VBM and CBM. An indirect band gap is found since the VBM is at the $\mathrm{K}$ point and the CBM lies at the $\Gamma$ point. The resulting indirect band gap of $1.0 \mathrm{eV}$ is larger than the planar and buckled bismuthene band gaps. This is similar to what has been reported in $\mathrm{H}$ and $\mathrm{CH}_{3}$ adsorbed on bismuthene using GGA calculations. $^{3,4,21}$ One can see that most $\mathrm{p}_{x}$ and $\mathrm{p}_{y}$ orbitals belonging to bismuth contribute to states close to the Fermi level. The CBM contains both $\mathrm{p}_{x}$ and $\mathrm{p}_{y}$ characters of the bismuth and the ligand. As a conclusion, in the full coverage regime, the hybrid systems contain significant contribution from both bismuth and ligand orbitals. However, the interaction between ligands at such a high coverage induces strain, which weakens the in-plane $\mathrm{Bi}-\mathrm{Bi}$ bonds. Although this effect makes the in-plane bonds longer, the bismuth-bismuth interactions remain strong enough to stabilize the hybrid layers. This will be discussed below, as there is a net charge accumulation of electrons on the ligand oxygen atom. We should mention that $s$ electrons do not contribute to states close to the Fermi level, since they appear far below in energy from the top of the valence band.

In addition to the projected band structure, we show the projected density of states (PDOS) on the individual atoms in Figure 4. Figure $4 \mathrm{a}$ shows the total (blue), s (magenta), $\mathrm{p}_{x}$ 

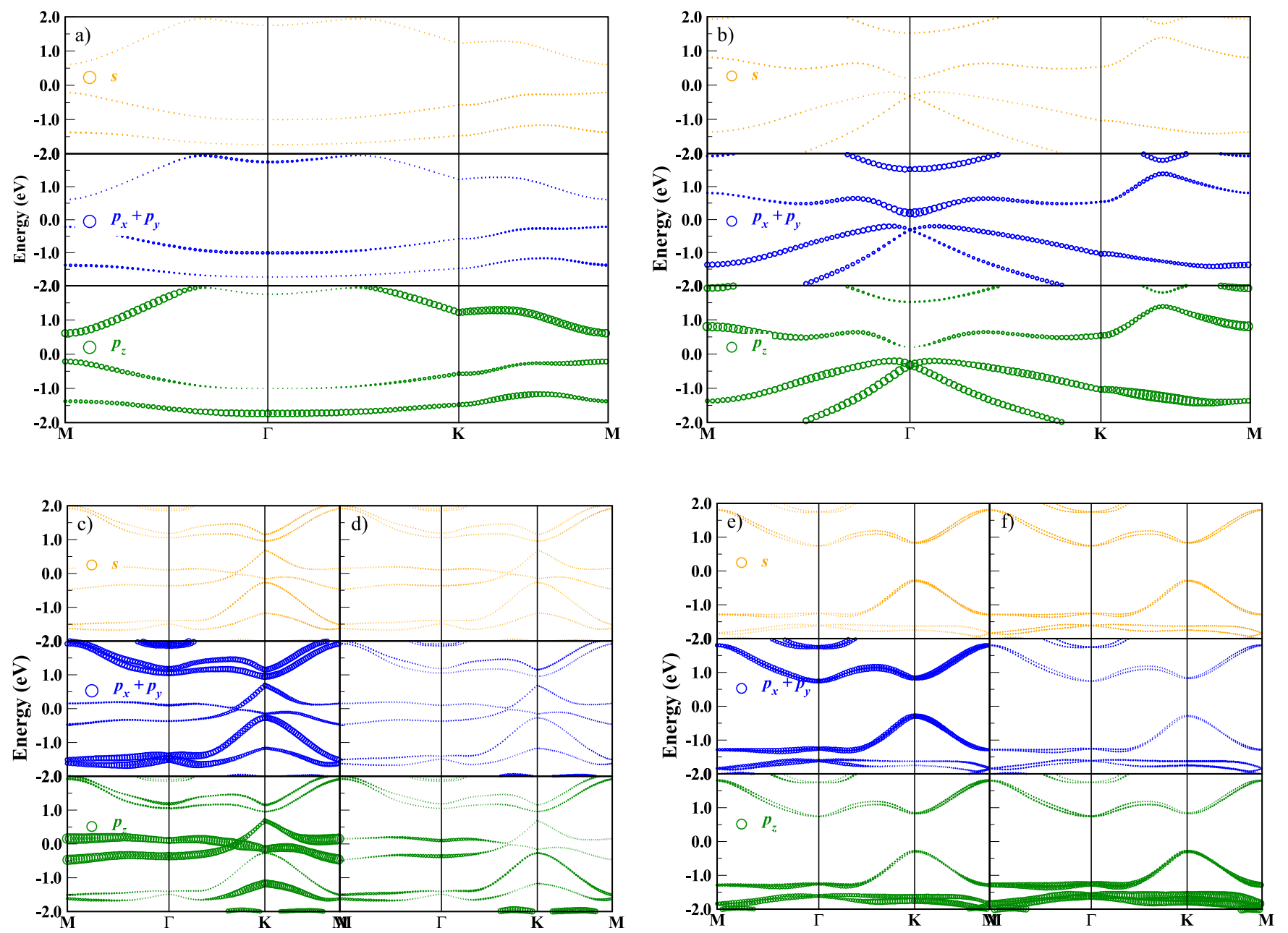

Figure 3. Projected band structures including SOC on $\mathrm{s}$ (orange), $\mathrm{p}_{x}+\mathrm{p}_{y}$ (blue), and $\mathrm{p}_{z}$ (green) orbitals for (a) planar bismuthene, (b) buckled bismuthene, and (c) and (d) 1/2 ML (one side adsorption) and (e) and (f) $1 \mathrm{ML}$ (two-side adsorption) of - COOH on bismuthene. The Fermi level is set to zero. The size of the spheres is proportional to the orbital contribution.

(cyan), $\mathrm{p}_{y}$ (red), and $\mathrm{p}_{z}$ (orange) orbitals of the bismuth atoms for planar bismuthene. Figure $4 \mathrm{~b}$ shows total, $\mathrm{s}, \mathrm{p}_{x}, \mathrm{p}_{y}$, and $\mathrm{p}_{z}$ orbitals of bismuth atoms for buckled bismuthene. Figure $4 \mathrm{c}$ shows the PDOS on $\mathrm{Bi}$ and ligand $\mathrm{C}$ and $\mathrm{O}$ atoms for $1 / \mathrm{ML}$ $-\mathrm{COOH}$ on bismuthene. One can see that the largest contributions are associated with $\mathrm{Bi}-\mathrm{p}_{z}$ localized orbitals. Figure $4 \mathrm{~d}$ shows the individual contributions for $1 \mathrm{ML}-\mathrm{COOH}$ on bismuthene.

The atomic orbital interpretation of the bands close to the Fermi level can be split into two main energetic range regions, namely, $\Phi_{1}$ and $\Phi_{2}$, shown in Figure 4e. We can see that the contribution of the carbon orbitals is very small compared to the bismuth and oxygen atoms in both $\Phi_{1}$ and $\Phi_{2}$ regions. Moreover, the $\sigma$-like bond between $\mathrm{Bi}-\mathrm{C}$ has a large contribution of the bismuth $\mathrm{p}_{z}$ orbitals with a large electron donation to carbons atoms. In this scenario, the bismuth atoms act as a Lewis base donating electrons through the $\sigma$ bond. However, the carbon atoms act as a Lewis acid receiving electrons from the bismuth atoms via the $\sigma$ bond.

The Bi-C $\sigma$-like bond is better represented by the $\Phi_{2}$ energy region shown in Figure $4 \mathrm{~d}$. The $\Phi_{1}$ region is mainly formed by bismuth-bismuth bonds that can be interpreted as a combination of the in-plane orbitals, $\mathrm{p}_{x}$ and $\mathrm{p}_{y}$. The $\Phi_{1}$ region contemplates the region close to the VBM, and so it dictates the band gap behavior. As we can see from the electronic charge density in Figure 4e, $\Phi_{1}$ comes mostly from the in-plane Bi-Bi $\left(\mathrm{p}_{x}, \mathrm{p}_{y}\right)$ bonds. So, the increase in the band gap of the $\mathrm{Bi}-$ $\mathrm{COOH}$ with respect to the buckled bismuthene must be due to the flattening of the $\mathrm{Bi}-\mathrm{Bi}$ bonds. The $\Phi_{2}$ region is mostly associated with a $\mathrm{Bi}-\mathrm{C} \sigma$-like bond, as it can be seen in Figure $4 a, b$. The relevant $\mathrm{Bi}-\mathrm{COOH}$ bands is basically formed by $\mathrm{Bi}-$ $\mathrm{Bi}$ bonds with delocalized orbitals, which can be explained by the composition of $\mathrm{p}_{x}$ and $\mathrm{p}_{y}$ Bi orbitals. Moreover, the $\mathrm{Bi}-\mathrm{C}$ bond lies deeper inside the valence band demonstrating in fact the presence of a $\mathrm{Bi}-\mathrm{C}$ bond.

The nature of the chemical bonds in the hybrid system was investigated by calculating the charge density difference between the electronic densities of $\mathrm{Bi}-\mathrm{COOH}$ and their constituent systems. The charge density difference plots require three calculations: the charge density of the hybrid system, the substrate (bismuthene), and the adsorbed system $(-\mathrm{COOH})$. This allows us to analyze the effect the $-\mathrm{COOH}$ interaction of the adsorbate with the substrate. The electronic density difference $\Delta \rho$ is given by $\Delta \rho=\rho^{\mathrm{Bi}-\mathrm{COOH}}-\rho^{\mathrm{Bi}}-\rho^{-\mathrm{COOH}}$, where $\rho^{\mathrm{Bi}-\mathrm{COOH}}$ is the electronic density of the hybrid $\mathrm{Bi}-$ $\mathrm{COOH}$ layers and $\rho^{\mathrm{Bi}}$ and $\rho^{-\mathrm{COOH}}$ are the charge densities calculated at fixed atomic positions of the $\mathrm{Bi}$ and $-\mathrm{COOH}$, respectively. The charge density difference shown in Figure 5 reflects how the electronic charge density changes upon $-\mathrm{COOH}$ adsorption. The hybrid $\mathrm{Bi}-\mathrm{COOH}$ system has a 

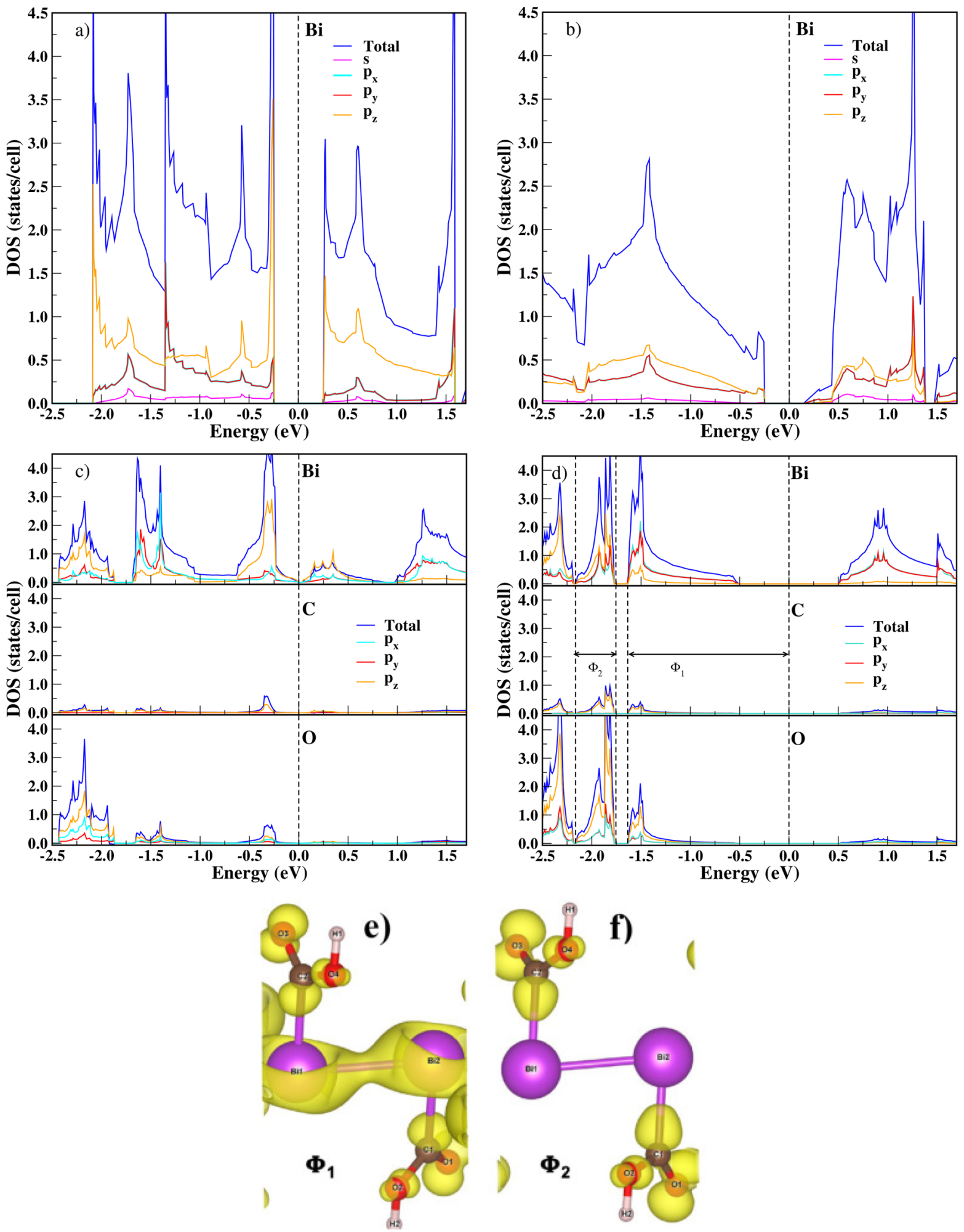

Figure 4. Orbital-resolved density of states for (a) planar bismuthene, (b) buckled bismuthene, (c) - COOH half-covered bismuthene, and (d) $-\mathrm{COOH}$ full-covered bismuthene. (e) and (f) show the total charge density of the regions $\Phi_{1}$ and $\Phi_{2}$. The Fermi level is set at zero.

larger electronic density between $\mathrm{Bi}-\mathrm{C}$ atoms. It is also seen in Figure 5 that the electronic charge density accumulates halfway in the bond regions, decreasing the electronic density around the atomic regions (shown in blue). The $\mathrm{Bi}-\mathrm{Bi}$ bond shows small changes, as can be seen in Figure $5 \mathrm{a}$ while a strong $\mathrm{Bi}-\mathrm{C}$ bond can be seen in Figure 5b. Since in buckled bismuthene the surface bismuth atoms are left with two extra electrons on the unsaturated bonds, upon $-\mathrm{COOH}$ adsorption covalent bonds between $\mathrm{C}$ and $\mathrm{Bi}$ bonds are expect to form. We can also notice that the charge is further distributed among the oxygen atoms on the ligand (namely $\mathrm{O} 1$ and O3). This is an important conclusion, since it reveals that the role of $-\mathrm{COOH}$ is not only to protect the $\mathrm{Bi}$ surface, but also to functionalize bismuthene by changing the reactivity of the system. 

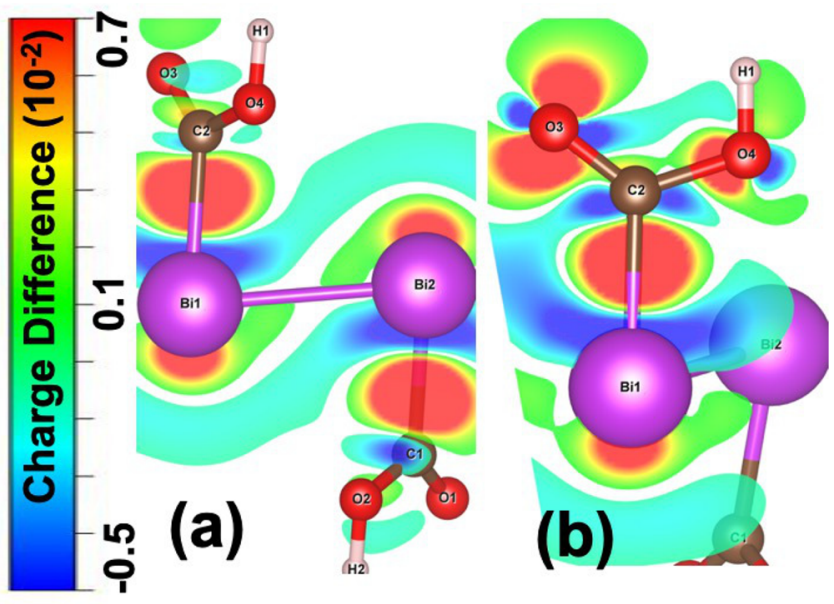

Figure 5. Charge density difference $(\Delta \rho)$ for monolayer $\mathrm{Bi}-\mathrm{COOH}$ in the $1 \mathrm{ML}$ regime. Negative (positive) values represent regions where electrons were accumulated (withdrawn) after adsorption. Cross sectional plots of (a) bismuth-bismuth bonds and (b) carbonoxygen-oxygen bonds.

In order to gain insight into the nature of the chemical bond environment in the functionalized $\mathrm{Bi}-\mathrm{COOH}$ system, the electronic localization function (ELF) ${ }^{22}$ was evaluated. ELF represents the electronic density localization in the spatial region of crystals and molecules, whose values vary between 0 and 1 . ELF values of about 0.5 can be interpreted as metallic-type (delocalized) bonds (green in Figure 6), whereas values close to

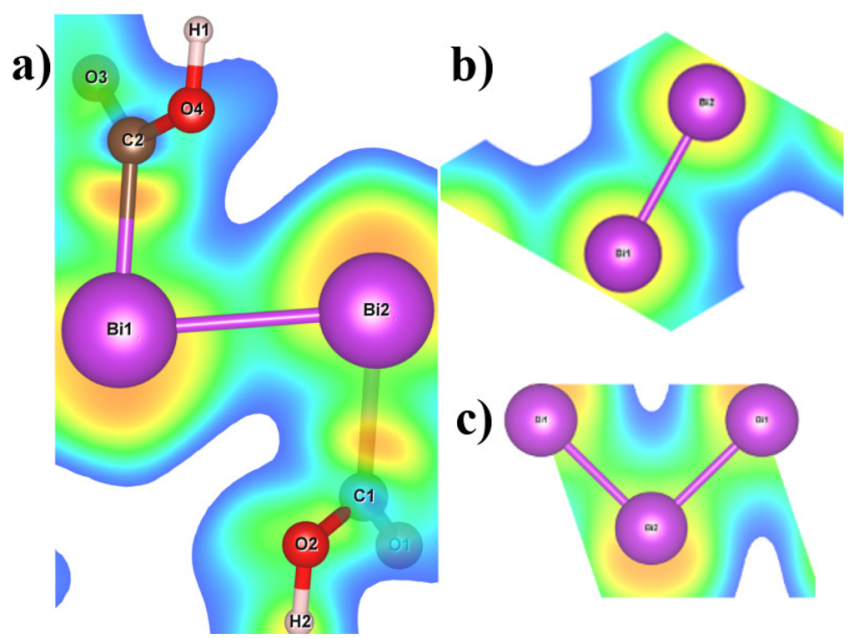

Figure 6. Electronic localization function cross-section for $\mathrm{Bi}-\mathrm{COOH}$ in the $1 \mathrm{ML}$ coverage regime: (a) cross section along the $\mathrm{Bi}-\mathrm{C}$ bonds; (b) and (c) top view of bismuth atoms only. The delocalized feature of bismuth bonds is shown in green. The localized regions due to covalent bonds and lone-pair electrons of nonbonding orbitals are shown in orange.

1.0 indicate localized electrons represented by covalent bonds between atoms or nonbonding lone electron pairs, shown in red in Figure 6). The ELF of the $\mathrm{Bi}-\mathrm{COOH}$, bibuckled and biplanar are presented in Figure 6. In Figure 6a, a region of localized electrons between carbon and bismuth atoms can be clearly identified (ELF values close to 1.0), thus characterizing a covalent bond. In addition, it is possible to see that the $\mathrm{C}-\mathrm{Bi}$ chemical bond has a slight ionic characteristic, which is usual for $-\mathrm{COOH}$ groups. In the bismuthene buckled structure, the ELF reveals a delocalized nature between Bi bonds, Figure $6 \mathrm{~b}$. The formation of the $\mathrm{Bi}-\mathrm{COOH}$ bonds induced a distortion into the geometry from a chairlike configuration in buckled bismuthene to a planar configuration. The chemical behavior of $-\mathrm{COOH}$ withdraws electronic charge of the bismuth atoms; this is contributing to the reduction of the electronic density between $\mathrm{Bi}-\mathrm{Bi}$ bonds. We can then conclude that the adsorption of $-\mathrm{COOH}$ groups not only changes the atomic structure of buckled bismuthene but also introduces a charge transfer between the ligand and bismuthene.

The question that rises now is whether the functionalized bismuthene has a topologically nontrivial band structure. We have calculated the $Z_{2}$ topological invariants according to ref 23 . This number corresponds to the number of Kramers pairs of edge modes, integrating over half of the Brillouin zone. If the overall $Z_{2}$ sum of occupied bands is even, the system is a regular insulator. If the sum is odd, the system is a topological insulator. In the $1 / 2 \mathrm{ML}$ coverage regime the hybrid $\mathrm{Bi}-\mathrm{COOH}$ system has a trivial band structure, and $Z_{2}$ is equal to 1 for $1 \mathrm{ML}$ coverage regime, which means that the hybrid $\mathrm{Bi}-\mathrm{COOH}$ layers behave as topological insulator. This confirms that we have calculated the band structure of a bismuth nanoribbon with zigzag edges adsorbed with $-\mathrm{COOH}$, as shown in Figure $7 \mathrm{a}$. Once the edges are exposed, it is possible to verify whether the topological states come from the edge atoms. The $\mathrm{Bi}-\mathrm{COOH}$ nanoribbons contain $28 \mathrm{Bi}$ atoms, $28 \mathrm{C}$ atoms, $56 \mathrm{O}$ atoms, and $28 \mathrm{H}$ atoms and have a width of $60 \AA$, which suffices to avoid spurious interaction between two edge ends (a ribbon with 102 Å width leads to identical results). Panels b and c of Figure 7 show the band projected electronic charge density at the $\Gamma$ point for the edge states. It is clearly seen that the spin up (Figure 7b) and spin down (Figure 7c) states are very localized at the edges only, confirming a nontrivial character of the band structure. The calculated electronic band structure of the functionalized nanoribbon along the ribbon growth direction is shown in Figure $7 \mathrm{~d}$. One can see the topological edge states with a single Dirac crossing at $\mathrm{M}$ points, confirming that the edge states are protected by time reversal symmetry.

Finally, we have computed the imaginary part of the electronic dielectric function $\left(\varepsilon_{2}\right)$ for the $\mathrm{Bi}-\mathrm{COOH}$ hybrid system within both the independent particle (IPA) and GW approximations, shown in Figure 8. The inclusion of SOC in the optical properties is also taken into account. Both IPA, Figure 8a, and $G W$, Figure $8 \mathrm{~b}$, approximations show an anisotropic behavior. In the IPA, an intense absorption band around $2.1 \mathrm{eV}$ is seen for the averaged $\left(\varepsilon_{x x}+\varepsilon_{y y}\right) / 2$ component, which is the response to light polarization along the bismuthene plane.

However, the $\varepsilon_{z z}$ component, which is the response to light polarization perpendicular to the bismuth layers, is broadly distributed in the region $0-10 \mathrm{eV}$. In the IPA, the electronic (indirect) band gap is $1.0 \mathrm{eV}$, which corresponds to the onset seen Figure 8a. The inclusion of many-body effects in the $G_{0} W_{0}$ approximation, i.e., without self-consistency either in the Green's function $G$ or in the screened Coulomb potential $W$ leads to a similar result and gives a value of $1.01 \mathrm{eV}$ for the electronic band gap. However, self-consistency in $G$ gives a value of $2.4 \mathrm{eV}$ to the band gap of the hybrid $\mathrm{Bi}-\mathrm{COOH}$, which is larger than the $G_{0} W_{0}$ value. This results are shown in Figure $8 \mathrm{~b}$. The first strong transition is around $3 \mathrm{eV}$. The $\varepsilon_{z z}$ component is a broad function between 5 and $30 \mathrm{eV}$. Our results show that the inclusion of many-body effects should give a better description of the screening properties. As a matter of comparison, in bismuthene, a band gap of $0.8 \mathrm{eV}$ is observed in bulk 
a)

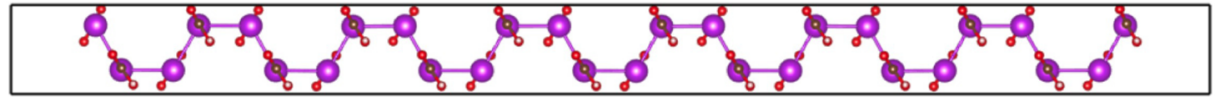

b)

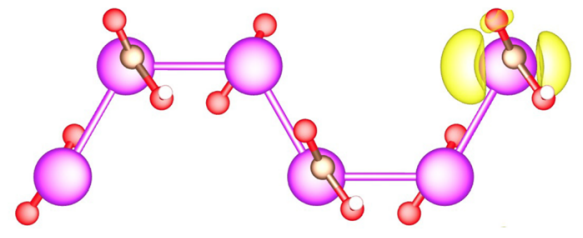

c)

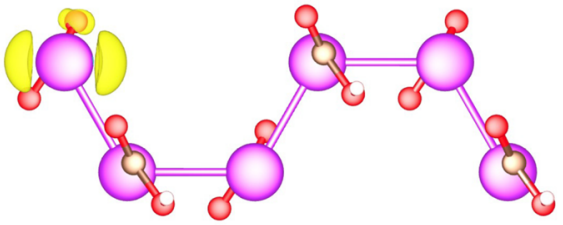

d)

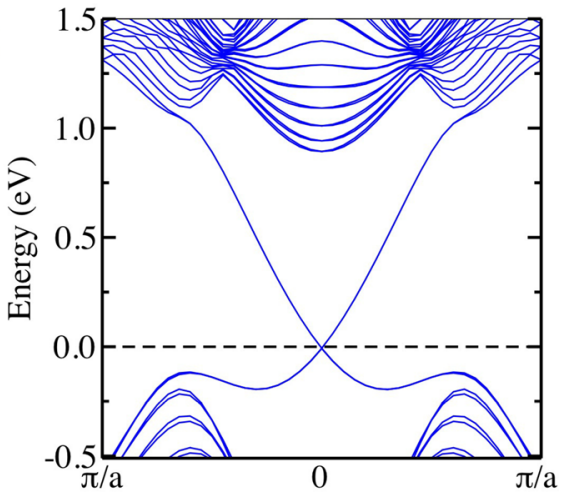

Figure 7. (a) DFT-GGA relaxed geometry of the $\mathrm{Bi}-\mathrm{COOH}$ nanoribbon, (b) band decomposed charge density at the $\Gamma$ point (spin up), (c) band decomposed charge density at the $\Gamma$ point (spin down), and (d) electronic band structure of a $\mathrm{Bi}-\mathrm{COOH}$ nanoribbon. The optimized in-plane lattice parameters are $a=5.5 \AA$ and $b=63.55 \AA$. The Fermi level is set at zero. The isosurface value is set to $0.002 \mathrm{e} / \AA^{3}$.

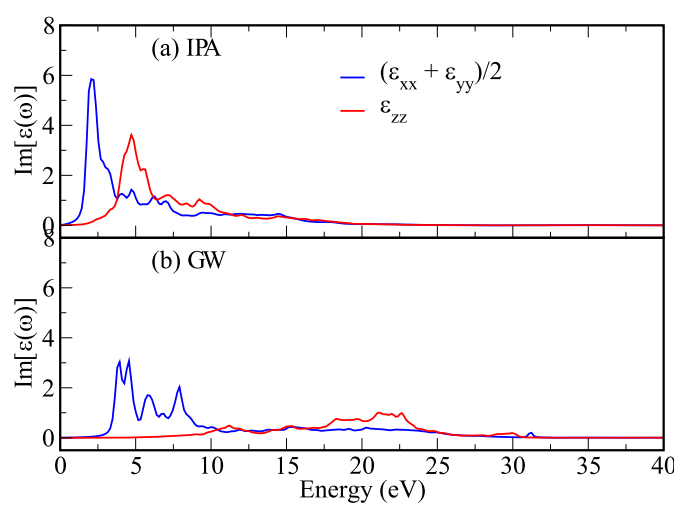

Figure 8. Imaginary electronic dielectric function $\left(\varepsilon_{2}\right)$ profile, for the $\mathrm{Bi}-\mathrm{COOH}$ system, in the (a) independent particle approximation (IPA) and (b) $G W$ approximation.

bismuthene. ${ }^{1}$ The DFT-GGA calculated value is $0.45 \mathrm{eV}$, whereas the computed value of the band gap with $G W$ is around $1.0 \mathrm{eV}^{21}$ Therefore, we can conclude that $G W$ provides a more realistic value for the CBM-VBM energy difference. Nevertheless, further experimental results are needed to validate our results.

\section{CONCLUSION}

We have performed density-functional theory and $G W$ calculations of electronic and dielectric properties for bismuthene adsorbed with $-\mathrm{COOH}$ groups. The electronic properties of this hybrid system show that the functionalized bismuthene in the full coverage regime has topological insulating behavior with a sizable band gap. Furthermore, the role of $-\mathrm{COOH}$ is not only to protect the $\mathrm{Bi}$ surface but also to functionalize bismuthene by changing the reativity of the system. This reactivity is desired for enhanced catalysis or immobilization of organic and biomolecules. However, ligands half-covered by bismuthene yield only a topologically trivial band gap. Finally, we show that the inclusion of many-body effects is very important to obtain a better description of the dielectric properties of the bismuthene hybrid system when compared to the independent particle approximation.

\section{AUTHOR INFORMATION}

\section{Corresponding Authors}

Andreia L. da Rosa - Institute of Physics, Federal University of Goiás, 74690-900 Goiânia, Goiás, Brazil; Bremen Center for Computational Materials Science, University of Bremen, 28359 Bremen, Germany; 이잉.org/0000-0002-2780-6448; Email: andreialuisa@ufg.br

Erika Nascimento Lima - Federal University of Rondonópolis, 78736-900 Rondonópolis, Mato Grosso, Brazil;

Email: erikanascimentolima@yahoo.com.br

Maurício Chagas da Silva - Bremen Center for Computational Materials Science, University of Bremen, 28359 Bremen, Germany; Max-Planck-Institute for the Structure and Dynamics of Matter, 22761 Hamburg, Germany; (1) orcid.org/00000002-6890-0182; Email: isobestico@gmail.com

Renato Borges Pontes - Institute of Physics, Federal University of Goiás, 74690-900 Goiânia, Goiás, Brazil; Email: pontes@ ufg.br

Jailton Souza de Almeida - Institute of Physics, Universidade Federal da Bahia, 40210-340 Salvador, Bahia, Brazil; Email: jailton.almeida@ufba.br

Tome Mauro Schmidt - Institute of Physics, Federal University of Uberlândia, 38400-902 Uberlândia, Minas Gerais, Brazil; 이이.org/0000-0002-9121-8157; Email: tschmidt@ufu.br

Thomas Frauenheim - Bremen Center for Computational Materials Science, University of Bremen, 28359 Bremen, Germany; Email: frauenheim@bccms.uni-bremen.de

Complete contact information is available at:

https://pubs.acs.org/10.1021/acs.jpcc.0c02385

\section{Notes}

The authors declare no competing financial interest.

\section{ACKNOWLEDGMENTS}

We acknowledge the financial support from the Brazilian Agencies CNPq and FAPEG (PRONEX 201710267000503) and German Science Foundation (DFG) under the program FOR1616. The calculations have been performed using the computational facilities of CENAPAD-SP and LNCC (Super- 
computer Santos Dumont) and at QM3 cluster at the Bremen Center for Computational Materials Science.

\section{REFERENCES}

(1) Reis, F.; Li, G.; Dudy, L.; Bauernfeind, M.; Glass; Hanke, W.; Thomale, R.; Schäfer, J.; Claessen, R. Bismuthene on a SiC substrate: A candidate for a high-temperature quantum spin Hall material. Science 2017, 357, 287-290.

(2) Yang, F.; Elnabawy, A. O.; Schimmenti, R.; Song, P.; Wang, J.; Peng, Z.; Yao, S.; Deng, R.; Song, S.; Lin, Y.; Mavrikakis, M.; Xu, W. Bismuthene for highly efficient carbon dioxide electroreduction reaction. Nat. Commun. 2020, 11, 1088.

(3) Ma, Y.; Dai, Y.; Kou, L.; Frauenheim, T.; Heine, T. Robust twodimensional topological insulators in methyl-functionalized bismuth, antimony, and lead bilayer films. Nano Lett. 2015, 15, 1083-1089.

(4) Freitas, R. R. Q.; Rivelino, R.; de Brito Mota, F.; de Castilho, C. M. C.; Kakanakova-Georgieva, A.; Gueorguiev, G. K. Topological Insulating Phases in Two-Dimensional Bismuth-Containing Single Layers Preserved by Hydrogenation. J. Phys. Chem. C 2015, 119, 23599-23606.

(5) Song, Z.; Liu, C.-C.; Yang, J.; Han, J.; Ye, M.; Fu, B.; Yang, Y.; Niu, Q.; Lu, J.; Yao, Y. Quantum spin Hall insulators and quantum valley Hall insulators of $\mathrm{BiX} / \mathrm{SbX}(\mathrm{X}=\mathrm{H}, \mathrm{F}, \mathrm{Cl}$ and $\mathrm{Br})$ monolayers with a record bulk band gap. NPG Asia Mater. 2014, 6, No. e147.

(6) Singh, S.; Zanolli, Z.; Amsler, M.; Belhadji, B.; Sofo, J. O.; Verstraete, M. J.; Romero, A. H. Low-Energy Phases of Bi Monolayer Predicted by Structure Search in Two Dimensions. J. Phys. Chem. Lett. 2019, 10, 7324.

(7) Liu, M.-Y.; Huang, Y.; Chen, Q.-Y.; Li, Z.-Y.; Cao, C.; He, Y. Strain and electric field tunable electronic structure of buckled bismuthene. RSC Adv. 2017, 7, 39546-39555.

(8) Kecik, D.; Özçlik, V. O.; Durgun, E.; Ciraci, S. Structure dependent optoelectronic properties of monolayer antimonene, bismuthene and their binary compound. Phys. Chem. Chem. Phys. 2019, 21, 7907-7917.

(9) Kou, L.; Fu, H.; Ma, Y.; Yan, B.; Liao, T.; Du, A.; Chen, C. Twodimensional ferroelectric topological insulators in functionalized atomically thin bismuth layers. Phys. Rev. B: Condens. Matter Mater. Phys. 2018, 97, No. 075429.

(10) Niu, C.; Bihlmayer, G.; Zhang, H.; Wortmann, D.; Blügel, S.; Mokrousov, Y. Functionalized bismuth films: Giant gap quantum spin Hall and valley-polarized quantum anomalous Hall states. Phys. Rev. B: Condens. Matter Mater. Phys. 2015, 91, 041303-041306.

(11) Blöchl, P. E. Projector augmented-wave method. Phys. Rev. B: Condens. Matter Mater. Phys. 1994, 50, 17953-17979.

(12) Kresse, G.; Joubert, D. From ultrasoft pseudopotentials to the projector augmented-wave method. Phys. Rev. B: Condens. Matter Mater. Phys. 1999, 59, 1758-1775.

(13) Perdew, J. P.; Burke, K.; Ernzerhof, M. Generalized Gradient Approximation Made Simple. Phys. Rev. Lett. 1996, 77, 3865-3868.

(14) Shishkin, M.; Marsman, M.; Kresse, G. Accurate Quasiparticle Spectra from Self-Consistent GW Calculations with Vertex Corrections. Phys. Rev. Lett. 2007, 99, 246403.

(15) Rasche, B.; Seifert, G.; Enyashin, A. Stability and Electronic Properties of Bismuth Nanotubes. J. Phys. Chem. C 2010, 114, 2209222097.

(16) Hirahara, T.; Fukui, N.; Shirasawa, T.; Yamada, M.; Aitani, M.; Miyazaki, H.; Matsunami, M.; Kimura, S.; Takahashi, T.; Hasegawa, S.; Kobayashi, K. Atomic and Electronic Structure of Ultrathin Bi(111) Films Grown on $\mathrm{Bi}_{2} \mathrm{Te}_{3}(111)$ Substrates: Evidence for a Strain-Induced Topological Phase Transition. Phys. Rev. Lett. 2012, 109, 227401.

(17) Cheng, L.; Liu, H.; Tan, X.; Zhang, J.; Wei, J.; Lv, H.; Shi, J.; Tang, X. Thermoelectric Properties of a Monolayer Bismuth. J. Phys. Chem. C 2014, 118, 904-910.

(18) Pumera, M.; Sofer, Z. 2D Monoelemental Arsenene, Antimonene, and Bismuthene: Beyond Black Phosphorus. Adv. Mater. 2017, 29, 1605299.
(19) Liu, M.-Y.; Chen, Q.-Y.; Cao, C.; He, Y. Topologically nontrivial phase and tunable Rashba effect in half-oxidized bismuthene. Phys. Chem. Chem. Phys. 2019, 21, 2899-2909.

(20) Jiang, S.; Butler, S.; Bianco, E.; Restrepo, O. D.; Windl, W.; Goldberger, J. E. Improving the stability and optical properties of germanane via one-step covalent methyl-termination. Nat. Commun. 2014, 5, 3389-3394.

(21) da Rosa, A. L.; Lima, E. N.; Pontes, R. B.; Schmidt, T.; Frauenheim, T. Tuning electronic and optical properties of bismuth monolayers by molecular adsorption. Submitted 2020.

(22) Becke, A. D.; Edgecombe, K. E. A simple measure of electron localization in atomic and molecular systems. J. Chem. Phys. 1990, 92, 5397-5403.

(23) Kane, C. L. Topological Band Theory and the Z2 Invariant. Contemporary Concepts of Condensed Matter Science 2013, 6, 3-34. 